\title{
Effects of Methotrexate on Transfected DNA Stability in Mammalian Cells
}

\author{
MARIA G. PALLAVICINI, ${ }^{1 *}$ PATRICIA S. DETERESA, ${ }^{1}$ CARIDAD ROSETTE,${ }^{1}$ JOE W. GRAY,${ }^{1}$ \\ AND FLORIAN M. WURM ${ }^{2}$ \\ Biomedical Sciences Division, L-452, Lawrence Livermore National Laboratory, Livermore, California $94550,{ }^{1}$ and
} Genentech, Inc., South San Francisco, California $94080^{2}$

Received 8 May 1989/Accepted 15 September 1989

\begin{abstract}
The chromosomal locations, amounts, and level of expression of transfected, amplified c-myc and dihydrofolate reductase sequences were measured in cells cultured in the presence and absence of methotrexate. These studies show that the location and amount of transfected sequences, as well as the level of expression, were more variable when the cells were cultured in methotrexate.
\end{abstract}

Mammalian cells transfected with specific DNA sequences produce recombinant proteins for biochemical and functional studies and therapeutic applications. Synthesis of recombinant proteins in transfected cells is directed by sequences that have integrated into the cellular genome $(1,2$, $6,8)$. Stability of the integrated sequence(s) is advantageous for these applications. Characterization of sequence integration is typically performed by Southern analysis. However, Southern analysis provides little information about heterogeneity in the amount(s) or integration sites of transfected sequences. These characteristics are likely to influence the stable production of recombinant proteins. We show that fluorescence in situ hybridization $(4,9,10)$ can provide information about integration heterogeneity. Specifically, we describe the use of in situ hybridization to characterize the pattern of incorporation of c-myc and dihydrofolate reductase (DHFR) sequences in a clonal cell line, HC-8, derived from methothrexate (MTX)-selected cells (CHO5AHS-Myc; 12) cotransfected with c-myc (under the control of an inducible Drosophila heat shock promoter) and DHFR. In addition, we quantitatively compare heterogeneity in the extent of c-myc amplification with heterogeneity in c-myc protein levels following heat induction of $\mathrm{HC}-8$ cells maintained in the presence and absence of MTX.

HC- 8 cells contain about 800 copies of murine c-myc sequence, whose expression is induced by exposure to elevated temperatures (12). These cells were cultured in selective culture medium with and without $250 \mu \mathrm{M}$ MTX (Sigma Chemical Co., St. Louis, Mo.) to determine the role of MTX in the maintenance of transfected sequences. Transcription of c-myc sequences was induced by replacing culture medium $\left(37^{\circ} \mathrm{C}\right)$ in $\mathrm{T}-75$ flasks containing $\mathrm{HC}-8$ cells with medium warmed to $43^{\circ} \mathrm{C}$ and submerging the flasks for $20 \mathrm{~min}$ in a circulating water bath maintained at $43^{\circ} \mathrm{C}$. Translation began $\sim 4 \mathrm{~h}$ after returning the heated flasks to a $\mathrm{CO}_{2}$ incubator at $37^{\circ} \mathrm{C}$.

Integration of recombinant sequences in cells cultured with and without MTX. Fluorescence in situ hybridization with a biotinylated c-myc DNA probe, pSP64myc3-2, which contains exons 2 and 3 of $c-m y c$ as well as plasmid sequences from pSP64 (12), coupled with detection using fluorescein isothiocyanate-labeled avidin (10) was used to study the chromosomal integration of transfected sequences homologous to the probe in metaphase spreads. Hybridization was

\footnotetext{
* Corresponding author.
}

also performed by using a probe for DHFR (12). In all cases, the DHFR integration pattern mimicked that of c-myc, so only c-myc hybridization patterns are shown. Figure $1 \mathrm{~A}$ shows the typical hybridization pattern of metaphases prepared from HC-8 cells cultured in MTX-free medium for an extended period. The transfected sequences in $\sim 99 \%$ of the cells were localized on the distal end of the long arm of chromosome 2 and comprised 10 to $15 \%$ of this chromosome. A few cells showed hybridization to two different chromosomes $(0.3$ to $0.6 \%)$ or to two regions on the same chromosome $(0.1 \%)$.

Transfer of the cells into medium containing MTX led to variability in the chromosomal location and amount of transfected sequences. Approximately $60 \%$ of the cells showed an integration pattern different from that in Fig. 1A. Figure $1 \mathrm{~B}$ through $\mathrm{F}$ shows several common hybridization patterns: (i) signal over 20 to $50 \%$ of one chromosome (C), (ii) multiple sites on one chromosome (E) and on multiple chromosomes (F), (iii) coverage of an entire ring chromosome (D), and (iv) signal on small derivative chromosomes or fragments (B).

Transfer of MTX-exposed cells back to medium lacking MTX resulted in reversion to the more homogeneous integration pattern on chromosome 2 . Figure 2 shows that the frequency of variant integration patterns decreased rapidly within 6 to 9 days after removal from medium containing MTX. Approximately $99 \%$ of the cells showed a single integration site on the distal end of chromosome 2 (Fig. 1A) within 8 to 22 days after removal of MTX.

Comparison of relative amounts of transfected c-myc sequences and c-myc protein levels. The relative amounts of transfected sequences present in cells maintained with and without MTX were estimated by analysis of the area covered by the hybridization probe by using a quantitative fluorescence image analysis system (7). Probe-linked fluorescence was excited at $480 \mathrm{~nm}$, and green fluorescence (500 to 540 nm) was imaged onto a camera. The camera output was digitized and transferred to a microVAX computer for processing. The total area covered by image elements whose intensity fell above a predetermined threshold was measured as an estimate of the amount of integrated c-myc sequence. Figure 3 shows the frequency distribution of hybridization areas for cells grown in the presence or absence of MTX. The average hybridized area in metaphases obtained from cells maintained for at least 60 days in MTX was threefold 

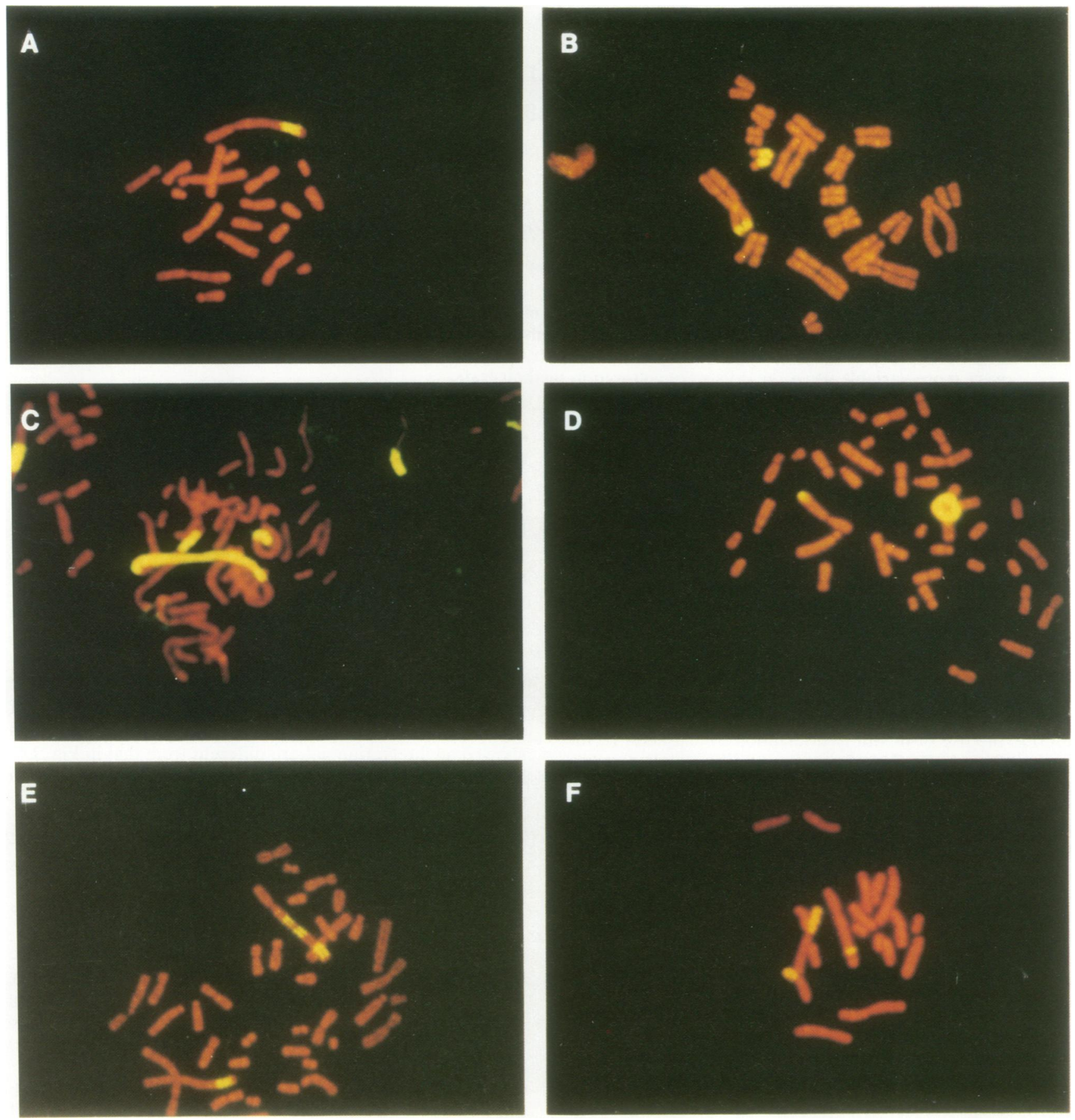

FIG. 1. Fluorescence in situ hybridization of metaphases from HC-8 cells cultured for more than 60 days without (A) and with (B through F) MTX. c-myc plasmid DNA was used for hybridization.

higher $\left(7.4\right.$ versus $\left.2.1 \mu \mathrm{m}^{2}\right)$ than that in cells grown without MTX.

Relative amounts of c-myc protein produced by cells cultured with and without MTX for at least 60 days were measured by flow cytometry following immunofluorescence staining with a mouse monoclonal antibody against c-myc (c-myc 152-6D11; NCI/BCB Repository, Microbiological Associates, Bethesda, Md.) and a fluorescein isothiocyanatelabeled goat anti-mouse antibody. Cells were harvested, fixed, and stained, as described elsewhere (C. Rosette,
P. D. DeTeresa, and M. G. Pallavicini, submitted for publication). Fluorescein isothiocyanate was excited at $488 \mathrm{~nm}$, and fluorescence was measured through a $514-\mathrm{nm}$ band pass filter. The immunofluorescence measurements were amplified logarithmically. Four separate experiments showed that protein-linked fluorescence intensity in MTX-treated cells was $3.2 \pm 1.6$ (standard deviation) times brighter than in cells cultured without MTX.

This study shows that HC-8 cells have integrated DHFR and c-myc sequences into one site at the distal end of the 


\section{C-MYC PROBE}

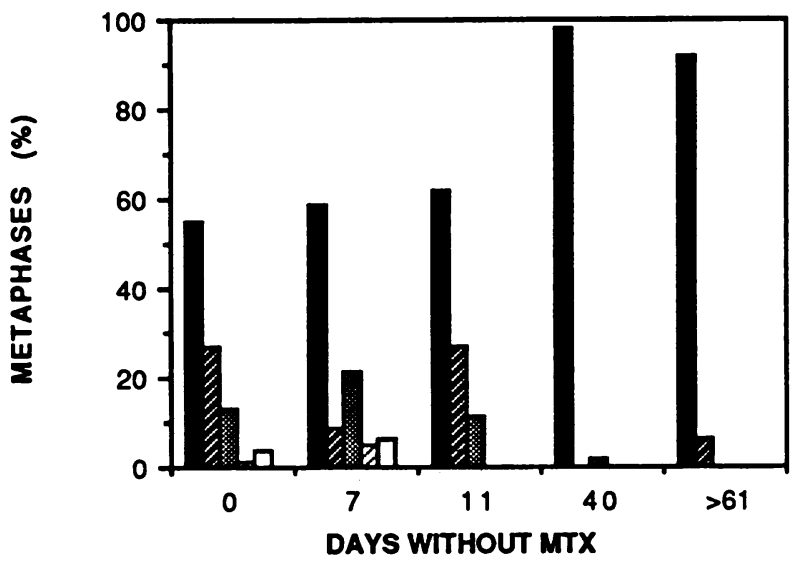

FIG. 2. Frequency of c-myc integration patterns observed in HC-8 cells maintained in MTX and in cells undergoing transition from selective to nonselective culture conditions. Metaphases (100 to 500) were analyzed for each time point. Frequency of integration(s): $\square$, one integration site per metaphase; $\mathbb{Z}$, signal over 20 to $50 \%$ of chromosome (one integration site per metaphase); integration sites per metaphase, each on different chromosomes; , two integration sites per metaphase, each on the same chromosome; $\square$, three integration sites on the same chromosome.

long arm of chromosome 2 and that this pattern is maintained stably in the absence of MTX selection. In fact, the stable pattern is disrupted by culture of the cells in MTX. Only $\sim 40 \%$ of cells grown in MTX show a pattern of c-myc incorporation in a single region on chromosome 2 , whereas the remainder show diverse integration patterns. Reversion to the stable integration pattern occurs rapidly after removal of MTX. Other studies have focused on the maintenance of constitutively expressed transfected sequences. Weidle et al. (11) reported a 60 to $70 \%$ loss of productivity of constitutively expressed tissue plasminogen activator within 50 days of MTX removal. Interestingly, tissue plasminogen activator production was stable for the remaining $\mathbf{5 0}$ days of the study. Kaufman et al. (3) and Michel et al. (5) reported clonal heterogeneity in the stability of the integration pattern of mammalian cells cotransfected with constitutively expressed tissue plasminogen activator or hepatitis $B$ virus DNA and DHFR sequences. Some cells lines maintained stable expression for more than 30 cell doublings in the absence of MTX, whereas in other clones, expression decreased significantly during the same time period.

Collectively, these data are consistent with the hypothesis that MTX is unnecessary for transfected sequence maintenance as long as the amplified sequence or its expression or both are not detrimental to the cells. An extreme example of detrimental effects of recombinant DNA-derived protein products is found in unsuccessful attempts to produce cell lines which constitutively overexpress c-myc protein $(R$. Kingston, personal communication). Thus, stability of integration in the absence of MTX selection may be heavily influenced by the toxicity of the protein product encoded by the transfected sequences. However, there may be cases in which continued exposure to MTX facilitates high expression of constitutively controlled sequences.

Data described in this paper also provide some insight into the mechanisms by which MTX leads to amplification of transfected sequences. Quantitative analysis of hybridization patterns showed substantial heterogeneity in cells grown in MTX-containing culture medium. On average, MTX-treated cells contained $\sim 3$ times more integrated c-

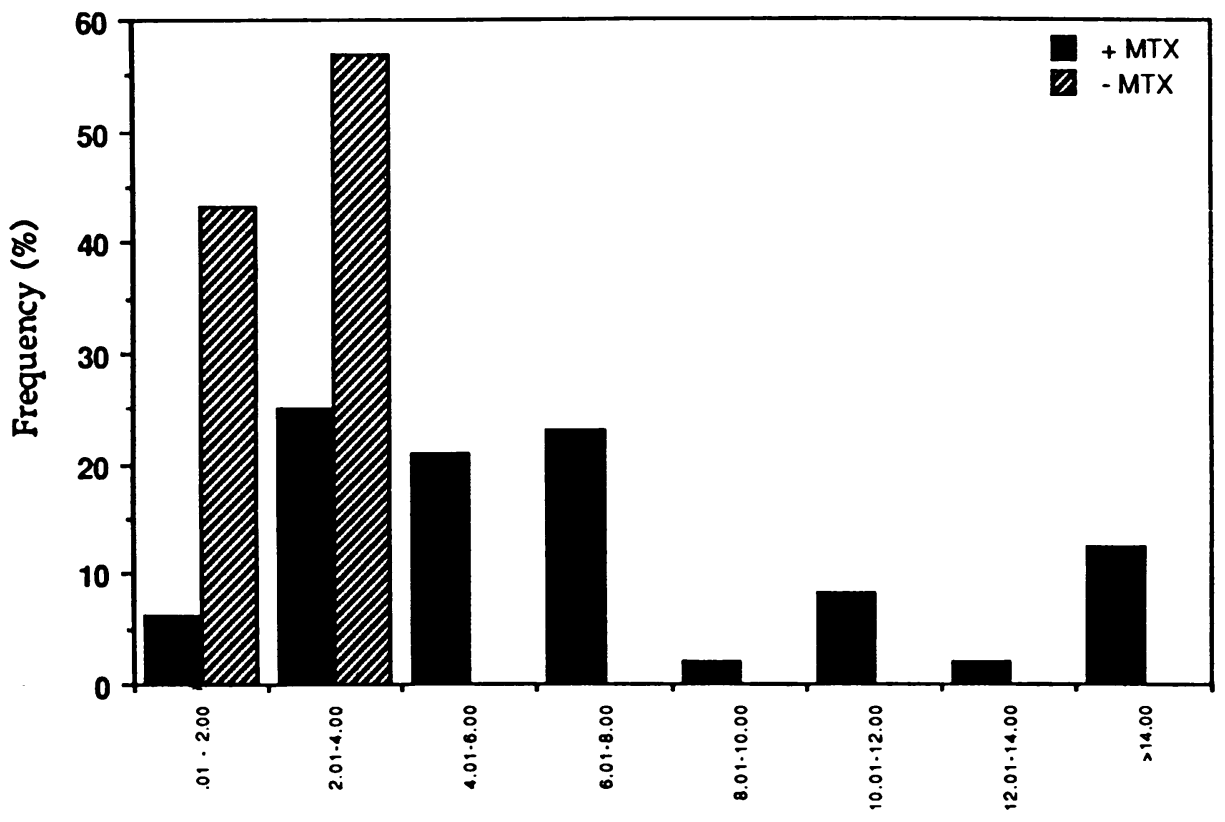

Area $\left(\mu \mathrm{m}^{2}\right)$ of Hybridized Region/Metaphase

FIG. 3. Quantitation of the relative amounts of transfected sequences contained in HC-8 cells maintained with and without MTX. Metaphases were obtained from HC-8 cells maintained continuously in the absence or presence of MTX for 60 days. Following hybridization with pSP64myc3-2, the areas corresponding to hybridized regions were measured by using quantitative fluorescence microscopy. A total of 48 and 65 metaphases were scored for cultures grown in the presence and absence of MTX, respectively. 
myc sequences than cells cultured without MTX. Protein production was higher by about the same amount in cells cultured in the presence of MTX. Thus, all amplified sequences appeared to be fully functional. We suggest that the increase in the average amount of transfected sequences may be due to increased heterogeneity in the integration pattern produced by growth in MTX coupled with the loss of cells carrying too little DHFR to allow propagation in MTX. Interestingly, most of the cells containing highly elevated amounts of transfected sequence were not stable and were lost in the absence of MTX. This suggests that the generation of a clone containing increased amounts of transfected sequences and having the ability to proliferate readily in the absence of MTX (compared to original cells) is a rare occurrence. The mechanism that leads to stability in the absence of MTX remains unclear.

This work was performed under the auspices of the U.S. Department of Energy by the Lawrence Livermore National Laboratory under contract W-7405-ENG-48.

\section{LITERATURE CITED}

1. Kaufman, R. J., and P. A. Sharp. 1982. Amplification and expression of sequences cotransfected with modular dihydrofolate reductase cDNA gene. Mol. Biol. 159:601-621.

2. Kaufman, R. J., P. A. Sharp, and S. A. Latt. 1983. Evolution of chromosomal regions containing transfected and amplified dihydrofolate reductase sequences. Mol. Cell. Biol. 3:699-711.

3. Kaufman, R. J., L. C. Wasley, A. J. Spiliotes, S. D. Gossels, S. A. Latt, G. R. Larsen, and R. M. Kay. 1985. Coamplification and coexpression of human tissue-type plasminogen activator and murine dihydrofolate reductase sequences in Chinese hamster ovary cells. Mol. Cell. Biol. 5:1750-1759.
4. Lawrence, J. B., C. A. Villnave, and R. H. Singer. 1988. Sensitive, high resolution chromatin and chromosome mapping in situ: presence and orientation of two closely related copies of EBV in a lymphoma line. Cell 52:51-61.

5. Michel, M. L., E. Sobczak, Y. Mapliece, P. Tiollais, and R. E. Streek. 1985. Expression of amplified hepatitis B virus surface antigen genes in Chinese hamster ovary cells. Bio/Technology 3:561-566.

6. Milbrandt, J. D., J. C. Azizkhan, and J. L. Hamlin. 1983. Amplification of a cloned Chinese hamster dihydrofolate reductase gene after transfer into a dihydrofolate reductase-deficient cell line. Mol. Cell. Biol. 3:1274-1282.

7. Mullikin, J., J. W. Gray, D. Pinkel, B. Trask, and H. Van Dekken. 1987. System configuration for mapping architecture of human nuclei. Cytometry Suppl. 1:8.

8. Pallavicini, M. G., P. S. DeTeresa, and F. M. Wurm. 1988. Use of fluorescence in situ hybridization to detect and monitor transfected and amplified sequences in recombinant $\mathrm{CHO}$ cells. Dev. Biol. Stand. 70:165-172.

9. Pinkel, D., J. Landegent, C. Collins, J. Fuscoe, R. Seagraves, J. Lucas, and J. Gray. 1988. Fluorescence in situ hybridization with human chromosome specific libraries: detection of Trisomy 21 and translocations of chromosome 4. Proc. Natl. Acad. Sci. USA 85:9138-9142.

10. Pinkel, D., T. Straume, and J. W. Gray. 1986. Cytogenetic analysis using quantitative, high sensitivity fluorescence hybridization. Proc. Natl. Acad. Sci. USA 83:2934-2938.

11. Weidle, U. H., P. Buckel, and J. Wienberg. 1988. Amplified expression constructs for human tissue-type plasminogen activator in Chinese hamster ovary cells: instability in the absence of selective pressure. Gene 66:193-203.

12. Wurm, F. M., K. A. Gwinn, and R. E. Kingston. 1986. Inducible overproduction of the mouse c-myc protein in mammalian cells. Proc. Natl. Acad. Sci. USA 83:5414-5418. 